\title{
Generalized additive mixed model to evaluate the association between total pulmonary infection volume and volume ratio, and clinical types, in patients with COVID-19 pneumonia: a propensity score analysis
}

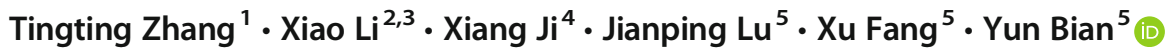 \\ Received: 1 October 2020 / Revised: 20 January 2021 / Accepted: 10 March 2021 / Published online: 15 April 2021 \\ (C) European Society of Radiology 2021
}

\begin{abstract}
Objectives To investigate the association between longitudinal total pulmonary infection volume and volume ratio over time and clinical types in COVID-19 pneumonia patients.

Methods This retrospective review included 367 patients with COVID-19 pneumonia. All patients underwent CT examination at baseline and/or one or more follow-up CT. Patients were categorized into two clinical types (moderate and severe groups). The severe patients were matched to the moderate patients via propensity scores (PS). The association between total pulmonary infection volume and volume ratio and clinical types was analyzed using a generalized additive mixed model (GAMM).

Results Two hundred and seven moderate patients and 160 severe patients were enrolled. The baseline clinical and imaging variables were balanced using PS analysis to avoid patient selection bias. After PS analysis, 172 pairs of moderate patients were allocated to the groups; there was no difference in the clinical and CT characteristics between the two groups $(p>0.05)$. A total of 332 patients, including 396 CT scans, were assessed. The impact of total pulmonary infection volume and volume ratio with time was significantly affected by clinical types ( $p$ for interaction $=0.01$ and 0.01 , respectively) using GAMM. Total pulmonary infection volume and volume ratio of the severe group increased by $14.66 \mathrm{~cm}^{3}$ (95\% confidence interval [CI]: 3.92 to 25.40 ) and $0.45 \%$ (95\% CI: 0.13 to 0.77 ) every day, respectively, compared to that of the moderate group.

Conclusions Longitudinal total pulmonary infection volume and volume ratio are independently associated with the clinical types of COVID-19 pneumonia.

Key Points

- The impact of total pulmonary infection volume and volume ratio over time was significantly affected by the clinical types (p for interaction $=0.01$ and 0.01 , respectively) using the GAMM.

- Total pulmonary infection volume and volume ratio of the severe group increased by $14.66 \mathrm{~cm}^{3}$ (95\% CI: 3.92 to 25.40$)$ and 0.45\% (95\% CI: 0.13 to 0.77) every day, respectively, compared to that of the moderate group.
\end{abstract}

Keywords Coronavirus infections $\cdot$ Tomography, x-ray computed $\cdot$ Pneumonia, propensity scores

Abbreviations

BMI

Body mass index
CI

COPD
Confidence interval

Chronic obstructive pulmonary disease

Tingting Zhang and Xiao Li are the joint first authors in the paper.

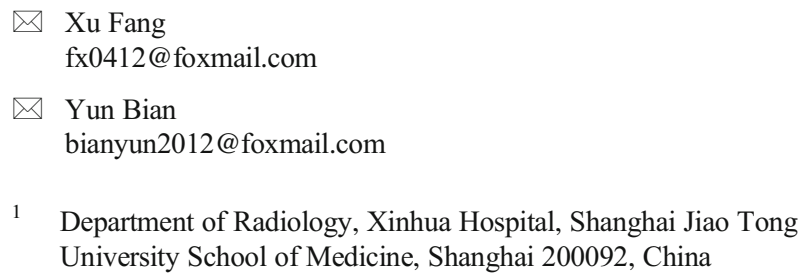

1 Department of Radiology, Xinhua Hospital, Shanghai Jiao Tong University School of Medicine, Shanghai 200092, China

2 Department of Medical Imaging, Jinling Hospital, Medical School of Nanjing University, Nanjing 210002, Jiangsu, China

3 Department of Radiology, Huoshenshan Hospital, Wuhan 430000, Hubei, China

4 Shanghai United Imaging Intelligence Healthcare, Shanghai, China

5 Department of Radiology, Changhai Hospital, The Navy Military Medical University, Changhai road 168, Shanghai 200434, China 


$\begin{array}{ll}\text { COVID-19 } & \text { Coronavirus disease } \\ \text { CRP } & \text { C-reactive protein } \\ \text { GAMM } & \text { Generalized additive mixed model } \\ \text { GGO } & \text { Ground glass opacity } \\ \text { HRCT } & \text { High-resolution CT } \\ \text { IL-6 } & \text { Interleukin-6 } \\ \text { MERS } & \text { Middle East respiratory syndrome } \\ \text { OR } & \text { Odds ratio } \\ \text { PS } & \text { Propensity scores } \\ \text { QCT } & \text { Quantitative computed tomography } \\ \text { SARS } & \text { Severe acute respiratory syndrome }\end{array}$

SARS-CoV-2 Severe acute respiratory syndrome coronavirus 2

VoD Volume of disease

WBC White blood cells

WHO World Health Organization

\section{Introduction}

In December 2019, a mass outbreak of a novel coronavirus infection occurred in Wuhan, Hubei Province, China. The novel coronavirus that was named severe acute respiratory syndrome coronavirus 2 (SARS-CoV-2) by the International Committee on Taxonomy of Viruses caused a pneumonia outbreak in China [1]. The disease caused by the virus, named coronavirus disease (COVID-19) by the World Health Organization (WHO), can spread through human-to-human contact [2]. At present, the infection has spread from China to the world, including more than 200 countries, reaching a pandemic level [3-5].

The National Health Commission of China formulated the Diagnosis and Treatment Program of 2019 New Coronavirus Pneumonia (seventh trial version) [6, 7], based on the recommendations of the WHO on severe acute respiratory syndrome (SARS) and the Middle East respiratory syndrome (MERS) $[8,9]$. In the seventh trial version, the clinical type of COVID-19 pneumonia was classified into mild, moderate, severe, and critical, according to the clinical manifestations [7]. Patients with mild and moderate disease have good prognosis. However, when COVID-19 pneumonia develops to severe and critical levels, pulmonary edema, respiratory failure, shock, and multiple organ failure can eventually cause death. Therefore, it is very important to accurately assess the factors related to disease severity in clinical practice. A previous study suggested that the total pulmonary infection volume was significantly associated with the clinical types of COVID-19 pneumonia [10], the decrease of the absolute value of CD4+T cells, and the increase of Interleukin-6 (IL-6) level [11]. Additionally, the total pulmonary infection volume can estimate the SARSCoV-2 burden [12] and accurately predict the need for oxygenation support and intubation [13]. The total pulmonary infection volume-based CT can be rapidly and accurately quantified using artificial intelligence (AI) technology. However, the published studies restrict analysis to the initial CT scan and do not focus on the changes of the total pulmonary infection volume over time. In addition, in clinical practice, it is hard to regularly follow up using CT, resulting in different time and frequency data. Generalized additive mixed models (GAMMs) [14] can easily accommodate unbalanced and unequally spaced observations [15] and are therefore ideal tools for analyzing longitudinal data. To the best of our knowledge, assessments of the association between longitudinal total pulmonary infection volume and volume ratio and clinical types in patients with COVID19 pneumonia have not been reported. Therefore, the primary objective of our study was to investigate the exact association between the longitudinal total pulmonary infection volume and volume ratio, and the clinical types in patients with COVID-19 pneumonia, using the GAMM.

\section{Materials and methods}

\section{Patients}

This retrospective cross-sectional study was reviewed and approved by the Biomedical Research Ethics Committee of our institution, and the requirement for patient consent was waived.

The inclusion criteria were as follows: (1) the availability of positive reverse-transcription polymerase chain reaction (RTPCR) tests confirming the SARS-Cov-2 origin of pneumonia, and (2) the availability of a chest $\mathrm{CT}$ at the time of diagnosis. We excluded the following patients: (1) patients with lung lesions that could not be visualized by high-resolution CT (HRCT) images, (2) patients with other lesions in the lung, and (3) patients with COVID-19 pneumonia in whom the interval between initial CT and confirmation of the clinical type was longer than 3 days. Finally, we retrospectively identified 367 consecutive patients with confirmed COVID-19 pneumonia at our Hospital between February 20 and March 31, 2020 (Fig. 1). All clinical results were extracted from their electronic medical records in the hospital information system. The mild type was defined as mild clinical symptoms with negative imaging findings. Thus, positive $\mathrm{CT}$ findings were often found in patients with moderate, severe, and critical disease [7] (Table 1). Therefore, the study participants were categorized into the following two groups: the moderate group and the severe group including severe and critical patients.

\section{CT scanning}

Pulmonary CT was performed using 128 -slice multidetector row CT scanners (uCT 760, United Imaging 


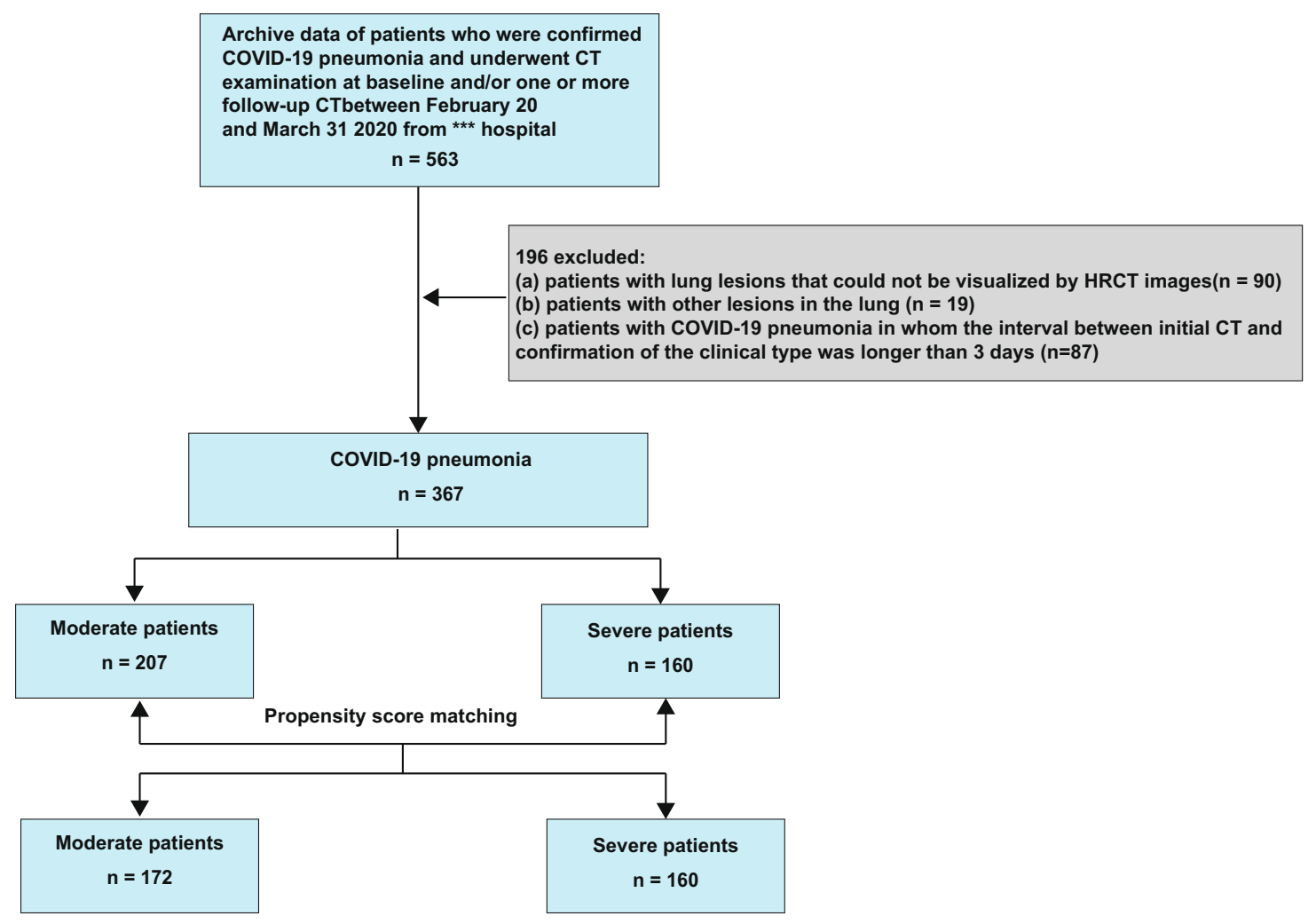

Fig. 1 Flow chart of the patient selection process

Healthcare). CT scans were obtained with the following parameters: $120 \mathrm{kV}$, adaptive tube current, beam collimation of $128 \times 0.6 \mathrm{~mm}$, and a $512 \times 512$ matrix. A nonenhanced CT was performed. The slice thickness was $0.625 \mathrm{~mm}$. Images were captured at window settings that allowed viewing of the lung parenchyma (window level 600 to $-700 \mathrm{HU}$; window width 1200-1500 HU) and the mediastinum (window level 20-40 HU; window width 400
$\mathrm{HU})$. The scanning range included all the regions of both lungs, from the apices to the bases.

\section{Radiological imaging analysis}

Total pulmonary infection volume and volume ratio were calculated by artificial intelligence software (uAI-Discover-NCP R001, United Imaging Healthcare) [16].
Table 1 The clinical classification of Covid-19 pneumonia from the 7 th edition of the National Commission of China classification [7]

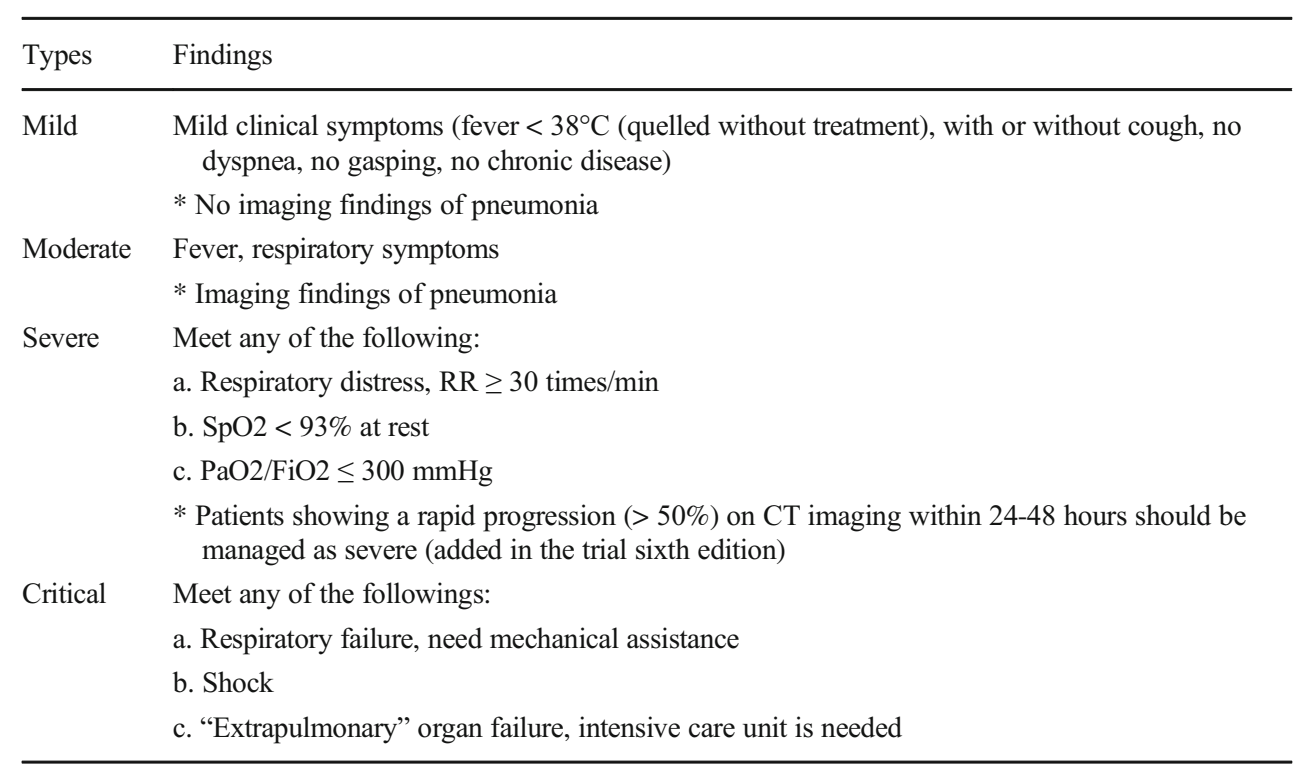

*CT findings 
We used the original cross-sectional images for analysis. All images were analyzed by two chest radiologists both with 8 years of experience, who were blinded to the clinical details. When their readings were not consistent, the final results were determined by consensus. The two chest radiologists evaluated the following characteristics: (a) location: right, left, or bilateral lungs; (b) distribution: peripheral, central, or diffuse (The outer one-third of the lung was defined as peripheral, the rest was defined as central [17], and diffuse included peripheral and central.); (c) attenuation: including ground glass opacity (GGO), crazy-paving pattern, and consolidation [18-20]; (d) maximum lesion range, only for the largest lesion: $\leq 5 \mathrm{~cm}, 5-10 \mathrm{~cm}$, and $>10 \mathrm{~cm}$; (e) lobe involvement: the five lung lobes were divided into categories of $\leq 2$ lobes, $3-4$ lobes, and 5 lobes; and (f) number of lesions: one, two, and three or more.

\section{Statistical analyses}

Normal distribution and variance homogeneity tests were performed on all continuous variables. Continuous variables (age, body mass index [BMI], total pulmonary infection volume, and volume ratio) with a normal distribution were expressed as mean \pm standard deviation; otherwise, they were expressed as median and range.

Study participants were categorized into the following two groups: the moderate group and the severe group. The severe patients were matched to the moderate patients via PS. A PS (logit model) was calculated for everyone, based on the baseline clinical variables (age, sex, smoking, body mass index, fever, cough, myalgia or arthralgia, headache, nausea or vomiting, diarrhea, abdominal pain, smoking history, cardiovascular disease, diabetes, hypertension, chronic obstructive pulmonary disease (COPD), chronic liver disease, chronic renal disease, malignant tumor, white blood cells (WBC) count, lymphocyte count, C-reactive protein (CRP), distribution, attenuation, maximum lesion range, number of lesions, and involvement of lobes). Matching was performed by using a 1:2 matching protocol to select the matched moderate group without replacement (greedy-matching algorithm), with a caliper width equal to 0.2 of the standard deviation of the logit of the PS. We compared the unmatched and matched baseline variables between the moderate and severe groups by using the chi-square test or Student $t$ test.

In this study, longitudinal data were the total pulmonary infection volume and volume ratio over time. The total pulmonary infection volume and ratio were repeatedly measured by AI software after every CT scan. We set the time of the initial CT scan as 0 , and the time was the interval from the initial CT scan. The longitudinal total pulmonary infection volume and volume ratio were analyzed using the GAMM [14], which easily accommodates unbalanced, unequally spaced observations [15], and is, therefore, an ideal tool for analyzing longitudinal data. All models also included intercept and time as random terms. Random effects allowed each participant's starting value to vary from the population average (intercept) and the longitudinal trajectory to vary from the population average longitudinal trajectory (slope). In the mixed-effects model, the interaction term between a fixed effect variable and time evaluates whether this variable is a predictor of the longitudinal changes in the dependent total pulmonary infection volume and volume ratio.

A two-tailed $p$ value less than 0.05 was considered statistically significant. All analyses were performed with the $\mathrm{R}$ software (version 3.4.3, R Foundation for Statistical Computing, and Empower Stats, X\&Y Solutions Inc.).

\section{Results}

\section{Clinical and CT characteristics before and after matching}

The clinical and CT baseline characteristics of the severe and moderate patients before and after PS matching are shown in Table 2. The PS distributions for the groups both before and after matching are shown in Fig. 2. Two hundred and seven moderate patients and 160 severe patients were enrolled. The baseline clinical and imaging variables were balanced using PS analysis to avoid patient selection bias. After PS analysis, 172 moderate patients matched with 160 severe patients were allocated to the groups and there was no statistically significant difference in the baseline clinical and CT characteristics between the two groups $(p>0.05)$. Before matching, age, cough, myalgia or arthralgia, distribution, attenuation, maximum lesion range, number of lesions, and the involvement of lobes were significantly different between the moderate and severe groups $(p<0.05)$. After matching, there was no statistically significant difference between the moderate and severe groups $(p>0.05)$. A total of 332 patients, including 396 CT scans, were assessed. All patients received supplemental oxygen therapy and antiviral therapy. Thirty-five severe patients received not only oxygen therapy and antiviral therapy but also hormone therapy. The CT scan frequency varied from 1 to 4 times (Table 3 ). The $\mathrm{CT}$ scan time was 3-31 days (mean days, 11.8 days) from the initial CT scan.

\section{Univariable analysis}

The baseline total pulmonary infection volume was 299.30 $\mathrm{cm}^{3}$ (range: $0.60-1973.50 \mathrm{~cm}^{3}$ ) in the moderate group and $658.65 \mathrm{~cm}^{3}$ (range: $2.60-1816.20 \mathrm{~cm}^{3}$ ) in the severe group. The baseline total pulmonary infection volume ratio was $7.75 \%$ (range: $0.01-52.20 \%$ ) in the moderate group and 
Table 2 The baseline clinical and CT characteristics of moderate and severe patients with COVID-19 pneumonia before and after propensity score analysis

\begin{tabular}{|c|c|c|c|c|c|c|}
\hline \multirow[t]{2}{*}{ Characteristics } & \multicolumn{3}{|l|}{ Before match } & \multicolumn{3}{|l|}{ After match } \\
\hline & Moderate $(n=207)$ & Severe $(n=160)$ & $p$ value & Moderate $(n=172)$ & Severe $(n=160)$ & $p$ value \\
\hline Sex, $n(\%)$ & & & 0.26 & & & 0.56 \\
\hline Male & $99(47.83)$ & $86(53.75)$ & & $87(50.58)$ & $86(53.75)$ & \\
\hline Female & $108(52.17)$ & $74(46.25)$ & & $85(49.42)$ & $74(46.25)$ & \\
\hline Age, mean (SD), years & $59.97 \pm 13.80$ & $64.83 \pm 12.86$ & $<0.001$ & $62.34 \pm 12.29$ & $64.83 \pm 12.86$ & 0.07 \\
\hline BMI, mean (SD), $\mathrm{kg} / \mathrm{m}^{2}$ & $23.02 \pm 3.32$ & $26.39 \pm 51.99$ & 0.35 & $23.08 \pm 3.51$ & $26.39 \pm 51.99$ & 0.41 \\
\hline Fever, $n(\%)$ & & & 0.19 & & & 0.79 \\
\hline No & $62(29.95)$ & $38(23.75)$ & & $43(25.00)$ & $38(23.75)$ & \\
\hline Yes & $145(70.05)$ & $122(76.25)$ & & $129(75.00)$ & $122(76.25)$ & \\
\hline Cough, $n(\%)$ & & & 0.03 & & & 0.25 \\
\hline No & $67(32.37)$ & $35(21.88)$ & & $47(27.33)$ & $35(21.88)$ & \\
\hline Yes & $140(67.63)$ & $125(78.12)$ & & $125(72.67)$ & $125(78.12)$ & \\
\hline Myalgia or arthralgia, $n(\%)$ & & & 0.09 & & & 0.46 \\
\hline No & $142(68.60)$ & $96(60.00)$ & & $110(63.95)$ & $96(60.00)$ & \\
\hline Yes & $65(31.40)$ & $64(40.00)$ & & $62(36.05)$ & $64(40.00)$ & \\
\hline Headache, $n(\%)$ & & & 0.24 & & & 1.00 \\
\hline No & $202(97.58)$ & $159(99.38)$ & & $170(98.84)$ & $159(99.38)$ & \\
\hline Yes & $5(2.42)$ & $1(0.62)$ & & $2(1.16)$ & $1(0.62)$ & \\
\hline Nausea or vomiting, $n(\%)$ & & & 1.00 & & & 1.00 \\
\hline No & $206(99.52)$ & $159(99.38)$ & & $171(99.42)$ & $159(99.38)$ & \\
\hline Yes & $1(0.48)$ & $1(0.62)$ & & $1(0.58)$ & $1(0.62)$ & \\
\hline Diarrhea, $n(\%)$ & & & 0.34 & & & 0.63 \\
\hline No & $199(96.14)$ & $150(93.75)$ & & $164(95.35)$ & $150(93.75)$ & \\
\hline Yes & $8(3.86)$ & $10(6.25)$ & & $8(4.65)$ & $10(6.25)$ & \\
\hline Abdominal pain, $n(\%)$ & & & 0.08 & & & 0.11 \\
\hline No & $207(100.00)$ & $157(98.12)$ & & $172(100.00)$ & $157(98.12)$ & \\
\hline Yes & 0 & $3(1.88)$ & & 0 & $3(1.88)$ & \\
\hline Smoking history, $n(\%)$ & & & 0.38 & & & 0.41 \\
\hline No & $195(94.20)$ & $147(91.88)$ & & $162(94.19)$ & $147(91.88)$ & \\
\hline Yes & $12(5.80)$ & $13(8.12)$ & & $10(5.81)$ & $13(8.12)$ & \\
\hline Cardiovascular disease, $n(\%)$ & & & 0.13 & & & 0.30 \\
\hline No & $179(86.47)$ & $129(80.62)$ & & $146(84.88)$ & $129(80.62)$ & \\
\hline Yes & $28(13.53)$ & $31(19.38)$ & & $26(15.12)$ & $31(19.38)$ & \\
\hline Diabetes, $n(\%)$ & & & 0.39 & & & 0.73 \\
\hline No & $168(81.16)$ & $124(77.50)$ & & $136(79.07)$ & $124(77.50)$ & \\
\hline Yes & $39(18.84)$ & $36(22.50)$ & & $36(20.93)$ & $36(22.50)$ & \\
\hline Hypertension, $n(\%)$ & & & 0.18 & & & 0.39 \\
\hline No & $137(66.18)$ & $95(59.38)$ & & $110(63.95)$ & $95(59.38)$ & \\
\hline Yes & $70(33.82)$ & $65(40.62)$ & & $62(36.05)$ & $65(40.62)$ & \\
\hline COPD, $n(\%)$ & & & 1.00 & & & 1.00 \\
\hline No & $204(98.55)$ & $157(98.12)$ & & $169(98.26)$ & $157(98.12)$ & \\
\hline Yes & $3(1.45)$ & $3(1.88)$ & & $3(1.74)$ & $3(1.88)$ & \\
\hline Chronic liver disease, $n(\%)$ & & & 0.78 & & & 0.77 \\
\hline No & $199(96.14)$ & $155(96.88)$ & & $165(95.93)$ & $155(96.88)$ & \\
\hline Yes & $8(3.86)$ & $5(3.12)$ & & $7(4.07)$ & $5(3.12)$ & \\
\hline Chronic renal disease, $n(\%)$ & & & 1.00 & & & 1.00 \\
\hline No & $201(97.10)$ & 155 (96.88) & & $166(96.51)$ & 155 (96.88) & \\
\hline
\end{tabular}


Table 2 (continued)

\begin{tabular}{|c|c|c|c|c|c|c|}
\hline \multirow[t]{2}{*}{ Characteristics } & \multicolumn{3}{|l|}{ Before match } & \multicolumn{3}{|l|}{ After match } \\
\hline & Moderate $(n=207)$ & Severe $(n=160)$ & $p$ value & Moderate $(n=172)$ & Severe $(n=160)$ & $p$ value \\
\hline Yes & $6(2.90)$ & $5(3.12)$ & & $6(3.49)$ & $5(3.12)$ & \\
\hline Malignant tumor, $n(\%)$ & & & 0.44 & & & 0.79 \\
\hline No & $200(96.62)$ & $152(95.00)$ & & $165(95.93)$ & $152(95.00)$ & \\
\hline Yes & $7(3.38)$ & $8(5.00)$ & & $7(4.07)$ & $8(5.00)$ & \\
\hline WBC count, $n(\%)$ & & & 0.56 & & & 0.91 \\
\hline Decreased & $9(4.35)$ & $4(2.50)$ & & $6(3.49)$ & $4(2.50)$ & \\
\hline Normal & $188(90.82)$ & $146(91.25)$ & & $156(90.70)$ & $146(91.25)$ & \\
\hline Increased & $10(4.83)$ & $10(6.25)$ & & $10(5.81)$ & $10(6.25)$ & \\
\hline Lymphocyte count, $n(\%)$ & & & 0.41 & & & 0.48 \\
\hline Increased & $32(15.46)$ & $33(20.62)$ & & $27(15.70)$ & $33(20.62)$ & \\
\hline Normal & $170(82.13)$ & $124(77.50)$ & & $140(81.40)$ & $124(77.50)$ & \\
\hline Decreased & $5(2.42)$ & $3(1.88)$ & & $5(2.91)$ & $3(1.88)$ & \\
\hline $\mathrm{CRP}, n(\%)$ & & & 0.59 & & & 0.53 \\
\hline Normal & $159(76.81)$ & $119(74.38)$ & & $133(77.33)$ & $119(74.38)$ & \\
\hline Increased & $48(23.19)$ & $41(25.62)$ & & $39(22.67)$ & $41(25.62)$ & \\
\hline Distribution, $n(\%)$ & & & $<0.001$ & & & 0.08 \\
\hline Peripheral or central & $87(42.03)$ & $39(24.38)$ & & $57(33.14)$ & $39(24.38)$ & \\
\hline Diffuse & $120(57.97)$ & $121(75.62)$ & & $115(66.86)$ & $121(75.62)$ & \\
\hline Attenuation, $n(\%)$ & & & 0.002 & & & 0.06 \\
\hline GGO and GGO + crazy-paving pattern & $70(33.82)$ & $39(24.38)$ & & $47(27.33)$ & $39(24.38)$ & \\
\hline GGO + consolidation & $39(18.84)$ & $16(10.00)$ & & $31(18.02)$ & $16(10.00)$ & \\
\hline GGO + crazy-paving pattern + consolidation & $98(47.34)$ & $105(65.62)$ & & $94(54.65)$ & $105(65.62)$ & \\
\hline Maximum lesion range, $n(\%)$ & & & 0.005 & & & 0.13 \\
\hline$\leq 5 \mathrm{~cm}$ & $74(35.75)$ & $36(22.50)$ & & 49 (28.49) & $36(22.50)$ & \\
\hline $5-10 \mathrm{~cm}$ & $64(30.92)$ & $46(28.75)$ & & $58(33.72)$ & $46(28.75)$ & \\
\hline$>10 \mathrm{~cm}$ & $69(33.33)$ & $78(48.75)$ & & $65(37.79)$ & $78(48.75)$ & \\
\hline Number of lesions, $n(\%)$ & & & 0.013 & & & 1.00 \\
\hline 1 & $8(3.86)$ & $2(1.25)$ & & $2(1.16)$ & $2(1.25)$ & \\
\hline 2 & $7(3.38)$ & 0 & & $1(0.58)$ & 0 & \\
\hline$\geq 3$ & $192(92.75)$ & $158(98.75)$ & & $169(98.26)$ & $158(98.75)$ & \\
\hline Involvement of lobes, $n(\%)$ & & & 0.004 & & & 0.60 \\
\hline 1 & $39(18.84)$ & $12(7.50)$ & & $14(8.14)$ & $12(7.50)$ & \\
\hline 2 & $96(46.38)$ & $75(46.88)$ & & $89(51.74)$ & $75(46.88)$ & \\
\hline 3 & $72(34.78)$ & $73(45.62)$ & & $69(40.12)$ & $73(45.62)$ & \\
\hline
\end{tabular}

Note: $B M I$, body mass index; $W B C$, white blood cell; $C R P$, C-reactive protein; $O R$, odds ratio; $C I$, confidence interval; $C O P D$, chronic obstructive pulmonary disease

20.95\% (range: $0.10-82.50 \%$ ) in the severe group (Fig. 3). The baseline total pulmonary infection volume and volume ratio were significantly different between the moderate and severe groups $(p<0.001)$ (Fig. 4). The univariable analysis showed that the baseline total pulmonary infection volume (odds ratio (OR): 1.00; 95\% confidence interval (CI): 1.00 to $1.00 ; p<0.0001)$ and volume ratio (OR: $1.05 ; 95 \% \mathrm{CI}$ : 1.03 to $1.07 ; p<0.0001$ ) were significantly associated with the clinical types (moderate and severe groups).

\section{Association between clinical types and longitudinal total pulmonary infection volume}

The GAMM showed that the total pulmonary infection volume did not significantly increase with time $(\beta: 242.86 ; 95 \%$ CI: -61.03 to $546.75 ; p=0.12$ ) in all the patients. Furthermore, subgroup analyses revealed that the total pulmonary infection volume did not significantly decrease with time ( $\beta$ : $-1.66 ; 95 \%$ CI: -10.82 to $7.50 ; p=0.73$ ) in the moderate 
Fig. 2 Propensity scores of the baseline characteristics before and after matching. a Dot plot of the distribution of propensity scores in either matched or unmatched groups. b Line plot of standardized differences before and after matching
Distribution of Propensity Scores
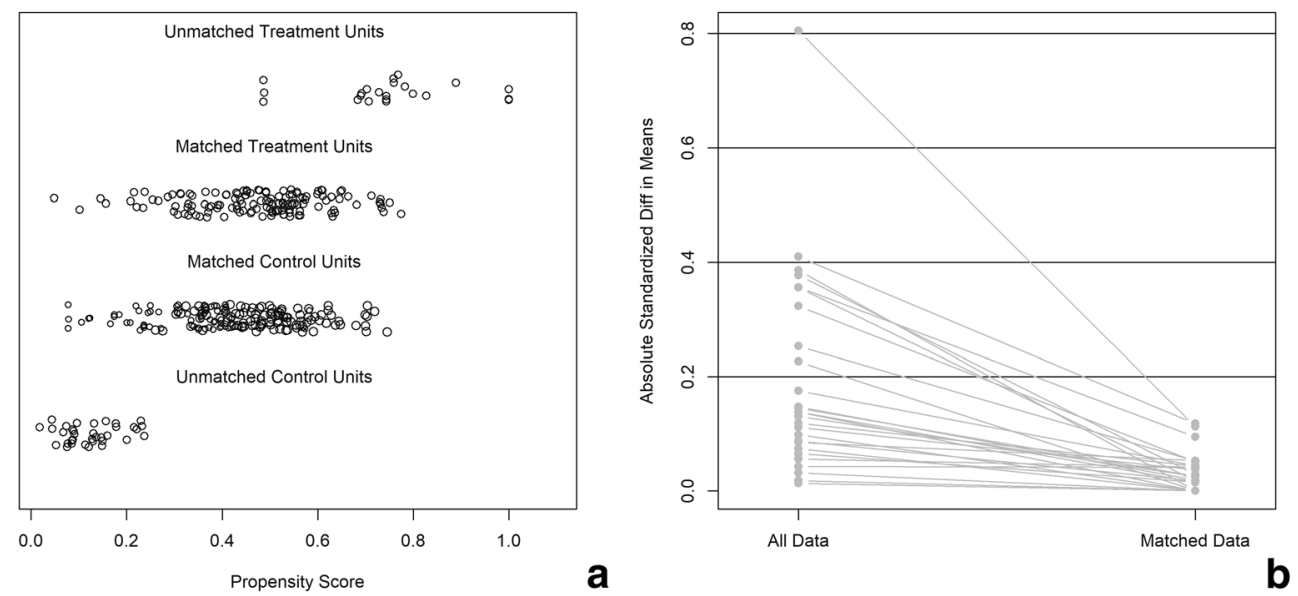

group. However, it significantly increased with time ( $\beta$ : 7.42; 95\% CI: 1.43 to $13.41 ; p=0.02$ ) in the severe group. The impact of total pulmonary infection volume with time was significantly affected by the clinical types ( $p$ for interaction $=0.01$ ). Total pulmonary infection volume of the severe group increased by $14.66 \mathrm{~cm}^{3}$ (95\% CI: 3.92 to 25.40 ) every day compared to that of the moderate group (Table 4 and Fig. 5).

\section{Association between clinical types and longitudinal total pulmonary infection volume ratio}

The GAMM model showed that the total pulmonary infection volume ratio increased significantly with time $(\beta$ : 11.79; $95 \%$ CI: 2.57 to $21.01 ; p=0.02$ ). Subgroup analyses revealed that the total pulmonary infection volume ratio did not significantly decrease with time $(\beta$ : $-0.04 ; 95 \% \mathrm{CI}$ : - 0.31 to $0.23 ; p=0.76$ ) in the moderate group. However, it significantly increased with time $(\beta: 0.19 ; 95 \% \mathrm{CI}: 0.02$ to $0.37 ; p=0.04)$ in the severe group. The impact of total pulmonary infection volume ratio with time was significantly affected by the clinical types $(\mathrm{p}$ for interaction $=$ $0.01)$. The total pulmonary infection volume ratio of the severe group increased by $0.45 \%$ (95\% CI: 0.13 to 0.77 ) every day compared to that of the moderate group (Table 4 and Fig. 5).

Table 3 CT follow-up frequencies

\begin{tabular}{lc}
\hline Scan frequency & $n(\%)$ \\
\hline 1 time & $281(71.0)$ \\
2 times & $40(10.1)$ \\
3 times & $9(2.3)$ \\
4 times & $2(0.5)$ \\
Total & $396(100.0)$ \\
\hline
\end{tabular}

\section{Discussion}

This study investigated the relationship between the longitudinal total pulmonary infection volume and volume ratio, and the clinical types of COVID-19 pneumonia, using the GAMM. Our results revealed that the impact of total pulmonary infection volume and volume ratio with time was significantly affected by the clinical types ( $p$ for interaction $=0.01$ and 0.01 , respectively) using the GAMM. Total pulmonary infection volume and volume ratio of the severe group increased by $14.66 \mathrm{~cm}^{3}$ (95\% CI: 3.92 to 25.40 ) and $0.45 \%$ ( $95 \%$ CI: 0.13 to 0.77 ) every day compared to that of the moderate group. Total pulmonary infection volume and volume ratio were independently associated with clinical types of COVID-19 pneumonia.

A few studies have explored the relationship between CT and clinical characteristics and disease severity. Li et al [21] found that the incidences of consolidation, linear opacities, crazy-paving pattern, and bronchial wall thickening were higher in severe/critical patients, while the incidences of lymph node enlargement, pericardial effusion, and pleural effusion were significantly higher in severe patients than those in ordinary patients. Zhao et al [22] found that architectural distortion, traction bronchiectasis, and CT involvement score according to the percentage of pneumonia involvement [23] aided in the evaluation of the severity and extent of the disease. Xiong et al [24] found that the highest temperature and severity of opacifications assessed on the initial CT were significantly related to the progression of opacifications on follow-up CT. Wang et al [25] found that the initial predominant pattern of abnormalities was ground glass opacity; the percent of mixed pattern peaked during illness days $12-17$ and became the second most prevalent pattern thereafter. Although these studies explored some CT and clinical characteristics 

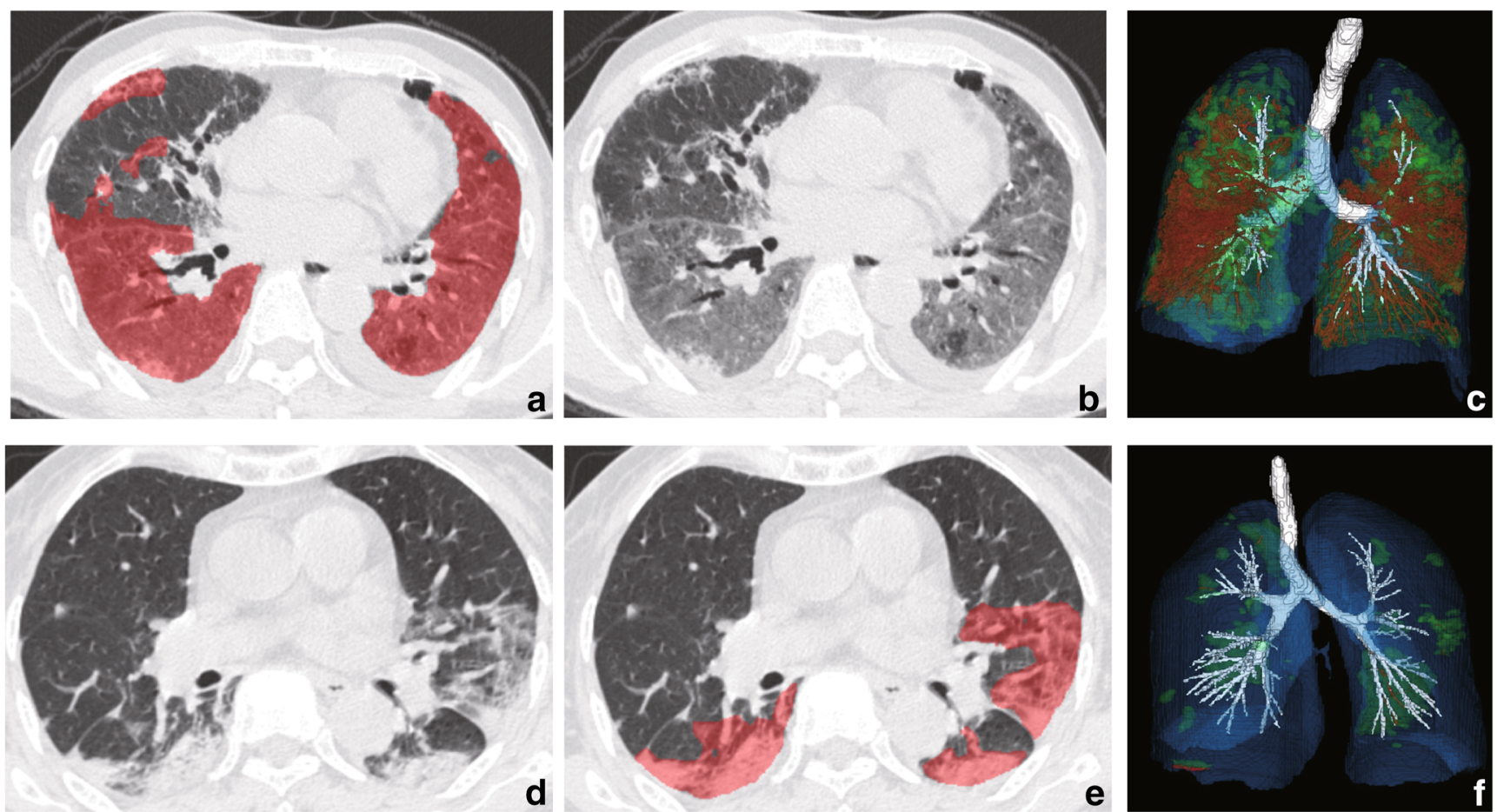

Fig. 3 a-c Chest CT findings of a 50-year-old man with severe COVID19 pneumonia (A) CT image of lung parenchyma showed multi-focal GGO, crazy-paving pattern, and consolidation peripherally distributed in the superior lobes of both lungs. b The lesions were automatically labeled by artificial intelligence software. $\mathbf{c}$ Three-dimensional volumerendered reconstruction showed the extent of GGO, crazy-paving pattern, and consolidation with a scattered pattern. $\mathbf{d}-\mathbf{f}$ Chest CT of a 28-year-old man with moderate COVID-19 pneumonia. d CT image of lung parenchyma showed multi-focal GGO and consolidation diffusely distributed in the middle and inferior lobes of the right lung and of the inferior lobe of the left lung. e The lesions were automatically labeled by artificial intelligence software. $\mathbf{f}$ Three-dimensional volume-rendered reconstruction showed the extent of the crazy-paving pattern and consolidation with a scattered pattern. GGO, ground glass opacity
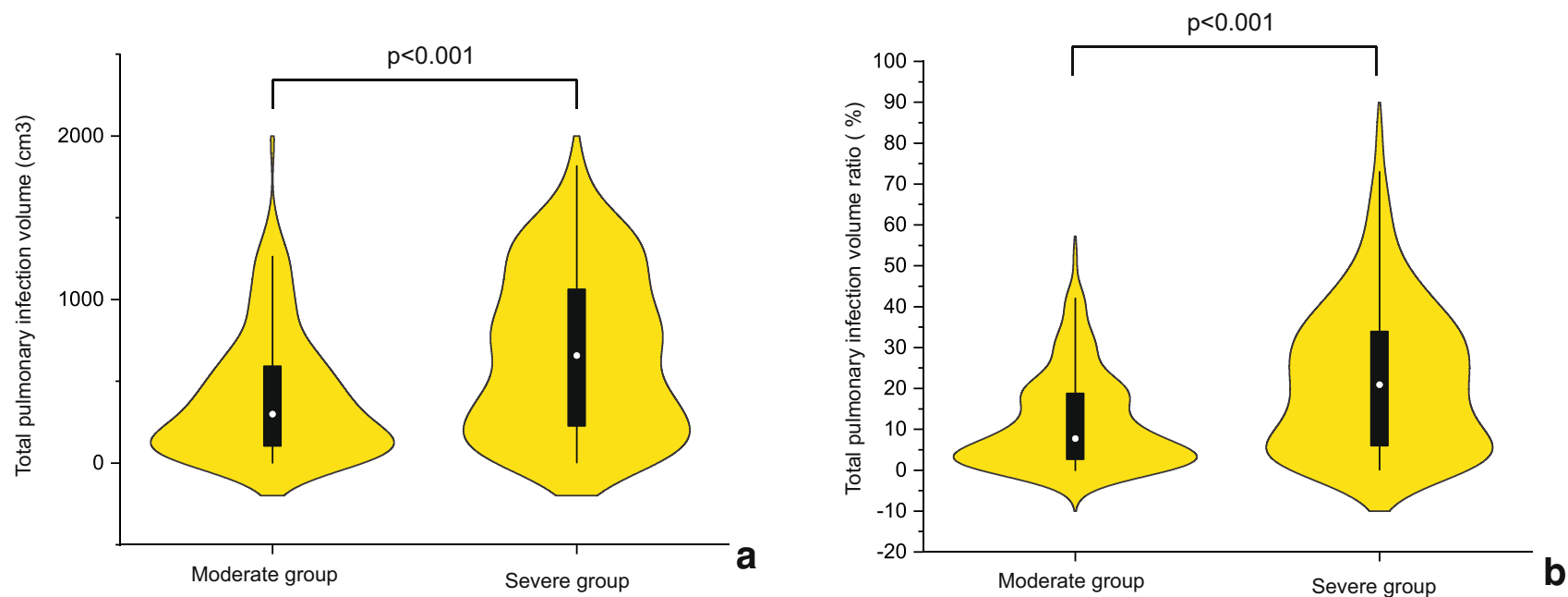

Fig. 4 Violin plot of total pulmonary infection volume and volume ratio. a Violin plot of total pulmonary infection volume between the moderate and severe groups. b Violin plot of total pulmonary infection volume ratio between the moderate and severe groups 
Table 4 Longitudinal total pulmonary infection volume and rate derived from a linear mixed-effects regression model

\begin{tabular}{|c|c|c|c|c|}
\hline & \multicolumn{2}{|c|}{ Total pulmonary infection volume $\left(\mathrm{cm}^{3}\right)$} & \multicolumn{2}{|c|}{ Total pulmonary infection volume ratio (\%) } \\
\hline & $\beta(95 \% \mathrm{CI})$ & $p$ value & $\beta(95 \% \mathrm{CI})$ & $p$ value \\
\hline Time (all patients) & $242.86(-61.03,546.75)$ & 0.12 & $11.79(2.57,21.01)$ & 0.02 \\
\hline Time (moderate group) & $-1.66(-10.82,7.50)$ & 0.73 & $-0.04(-0.31,0.23)$ & 0.76 \\
\hline Time (severe group) & $7.42(1.43,13.41)$ & 0.02 & $0.19(0.02,0.37)$ & 0.04 \\
\hline Time $\times$ severe vs moderate (test for interaction) & $14.66(3.92,25.40)$ & 0.01 & $0.45(0.13,0.77)$ & 0.01 \\
\hline
\end{tabular}

Note: $C I$, confidence interval

related to disease severity, they could not explore the relationship between computer-aided quantitative analysis CT characteristics and the severity of COVID-19.

At present, computer-aided quantitative analysis of quantitative computed tomography (QCT) has been used to diagnose COVID-19 pneumonia. Matos et al [12] quantified the volume of disease $(\mathrm{VoD})$ to predict the outcome and found that $\mathrm{VoD}$ was one of the important variables to predict severity. Lanza et al [13] tested QCT as an outcome predictor for COVID-19 and concluded that the compromised lung volume was the most accurate outcome predictor. He et al [11] studied the correlations of lymphocytes and cytokines between changes of lung lesion volumes in patients with COVID-19 and found that the decrease in the absolute value of $\mathrm{CD}^{+} \mathrm{T}$ cells and the increase in IL-6 levels significantly correlated with the volume of the lung lesion in critically ill patients. However, these studies did not take into account the volume of lesion changes with time. In this study, to avoid patient selection bias, the baseline clinical and imaging variables were balanced using PS matching. The GAMM model was used to accurately evaluate the relationship between total pulmonary infection volume and volume ratio and clinical type considering changes with time. The GAMM model showed that the impact of total pulmonary infection volume and volume ratio with time was significantly affected by the clinical types ( $p$ for interaction $=0.01$ and 0.01 , respectively). Total pulmonary infection volume and volume ratio of the severe group increased by $14.66 \mathrm{~cm}^{3}$ (95\% CI: 3.92 to 25.40$)$ and $0.45 \%$ (95\% CI: 0.13 to 0.77$)$ every day, compared to that of the moderate group.

However, our study had several limitations. Firstly, the results were established based on retrospective data obtained from a

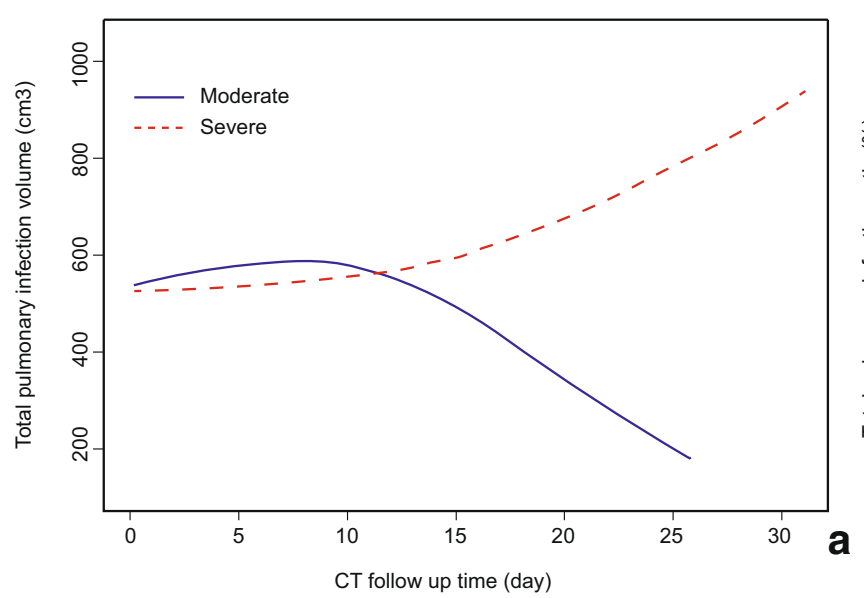

Fig. 5 Association between total pulmonary infection volume and volume ratio, and clinical types, using the generalized additive mixed model. a The impact of total pulmonary infection volume with time was significantly affected by the clinical types ( $p$ for interaction $=$ $0.01)$. Total pulmonary infection volume of the severe group increased by $14.66 \mathrm{~cm}^{3}$ (95\% CI: 3.92 to 25.40 ) every day, compared to that of the

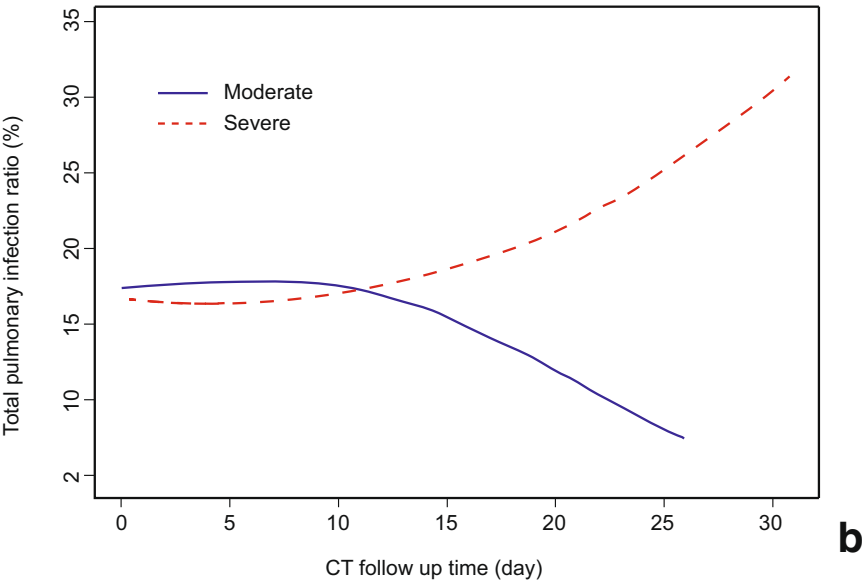

moderate group. b The impact of total pulmonary infection volume ratio with time was significantly affected by the clinical types ( $p$ for interaction $=0.01)$. The total pulmonary infection volume ratio of the severe group increased by $0.45 \%$ (95\% CI: 0.13 to 0.77 ) every day compared to that of the moderate group. GAMM, generalized additive mixed model; CI, confidence interval 
single center. Secondly, our study included only a small number of CT follow-up patients (332 patients, including 396 CT) and may not be sufficiently powered to detect changes in the total pulmonary infection volume. Thirdly, all patients received supplemental oxygen therapy and antiviral therapy in this study. So, we did not take into account the effect of treatment, which maybe lead to a bias. Lastly, we considered the most clinical and radiological characteristics from the published paper and our clinical experience for the PS analysis. But it did not mean that there are not more clinical and CT differences between the groups. This point also led to a bias. In the future, we will continue to follow up more patients to obtain more evidence for the clinical application of the lesion volume.

In conclusion, longitudinal total pulmonary infection volume and volume ratio are independently associated with the clinical types of COVID-19 pneumonia. This suggests that the changes in the total pulmonary infection volume and volume ratio with time could help to accurately predict severe and critical types of COVID-19 pneumonia.

Funding This work was supported in part by the National Science Foundation for Scientists of China (81871352) and National Science Foundation for Young Scientists of China (81701689)

\section{Declarations}

Guarantor The scientific guarantor of this publication is Yun Bian.

Conflict of interest The authors of this manuscript declare no relationships with any companies whose products or services may be related to the subject matter of the article.

Statistics and biometry No complex statistical methods were necessary for this paper.

Informed consent Written informed consent was waived by the Institutional Review Board.

Ethical approval Institutional Review Board approval was obtained by Huoshenshan Hospital.

\section{Methodology}

- Retrospective

- Diagnostic or prognostic study

- Performed at one institution

\section{References}

1. Sun P, Lu X, Xu C, Sun W, Pan B (2020) Understanding of COVID-19 based on current evidence. J Med Virol 92:548-551
2. Chan JF, Yuan S, Kok KH et al (2020) A familial cluster of pneumonia associated with the 2019 novel coronavirus indicating person-to-person transmission: a study of a family cluster. Lancet. https://doi.org/10.1016/S0140-6736(20)30154-9

3. Phan LT, Nguyen TV, Luong QC et al (2020) Importation and human-to-human transmission of a novel coronavirus in Vietnam. N Engl J Med 382:872-874

4. Holshue ML, DeBolt C, Lindquist S et al (2020) First case of 2019 novel coronavirus in the United States. N Engl J Med. https://doi. org/10.1056/NEJMoa2001191

5. Giovanetti M, Benvenuto D, Angeletti S, Ciccozzi M (2020) The first two cases of 2019-nCoV in Italy: where they come from? J Med Virol. https://doi.org/10.1002/jmv.25699

6. Organization WH Clinical management of severe acute respiratory infectionnwhen novel coronavirus $(2019-\mathrm{nCoV})$ infection is suspected. 2019. At https://www.who.int/csr/disease/coronavirus_ infections/InterimGuidance ClinicalManagement NovelCoronavirus_11Feb13u.pdf?ua $=1 \& u a=1$. Accessed February 11, 2020

7. General Office of National Health Committee. Office of State Administration of Traditional Chinese Medicine. Notice on the issuance of a program for the diagnosis and treatment of novel coronavirus (2019-nCoV) infected pneumonia (trial seventh edition). http://bgs.satcm.gov.cn/zhengcewenjian/2020-03-04/ 13594.html. Accessed 2020-03-04

8. Organization WH WHO guidelines for the global surveillance of severe acute respiratory syndrome (SARS). 2004. At https://www. who.int/csr/resources/publications/WHO_CDS_CSR_ARO 2004_1.pdf?ua $=1$. Published January, 2004. Updated October, 2004

9. Azhar EI, El-Kafrawy SA, Farraj SA et al (2014) Evidence for camel-to-human transmission of MERS coronavirus. N Engl J Med 370:2499-2505

10. Fang X, Li X, Bian Y, Ji X, Lu J (2020) Relationship between clinical types and radiological subgroups defined by latent class analysis in 2019 novel coronavirus pneumonia caused by SARSCoV-2. Eur Radiol. https://doi.org/10.1007/s00330-020-06973-9

11. He S, Zhou C, Lu D et al (2020) Relationship between Chest CT manifestations and immune response in COVID-19 patients. Int J Infect Dis. https://doi.org/10.1016/j.ijid.2020.06.059

12. Matos J, Paparo F, Mussetto I et al (2020) Evaluation of novel coronavirus disease (COVID-19) using quantitative lung CT and clinical data: prediction of short-term outcome. Eur Radiol Exp 4: 39

13. Lanza E, Muglia R, Bolengo I et al (2020) Quantitative chest CT analysis in COVID-19 to predict the need for oxygenation support and intubation. Eur Radiol. https://doi.org/10.1007/s00330-02007013-2

14. Geert Verbeke GM (2000) Linear mixed models for longitudinal data. Springer, New York

15. Gueorguieva R, Krystal JH (2004) Move over ANOVA: progress in analyzing repeated-measures data and its reflection in papers published in the Archives of General Psychiatry. Arch Gen Psychiatry 61:310-317

16. Ouyang X, Huo J, Xia L et al (2020) Dual-sampling attention network for diagnosis of COVID-19 from community acquired pneumonia. IEEE Trans Med Imaging 39:2595-2605

17. Song F, Shi N, Shan F et al (2020) Emerging 2019 Novel Coronavirus (2019-nCoV) Pneumonia. Radiology 295:210-217

18. Franquet $\mathrm{T}$ (2011) Imaging of pulmonary viral pneumonia. Radiology 260:18-39 
19. Koo HJ, Lim S, Choe J, Choi SH, Sung H, Do KH (2018) Radiographic and CT features of viral pneumonia. Radiographics 38:719-739

20. Hansell DM, Bankier AA, MacMahon H, McLoud TC, Muller NL, Remy J (2008) Fleischner Society: glossary of terms for thoracic imaging. Radiology 246:697-722

21. Li K, Wu J, Wu F et al (2020) The clinical and chest CT features associated with severe and critical COVID-19 pneumonia. Invest Radiol. https://doi.org/10.1097/RLI.0000000000000672

22. Zhao W, Zhong Z, Xie X, Yu Q, Liu J (2020) Relation between chest $\mathrm{CT}$ findings and clinical conditions of coronavirus disease (COVID-19) pneumonia: a multicenter study. AJR Am J Roentgenol 214:1072-1077

23. Xie X, Zhong Z, Zhao W, Zheng C, Wang F, Liu J (2020) Chest CT for typical 2019-nCoV pneumonia: relationship to negative RT-
PCR testing. Radiology. https://doi.org/10.1148/radiol. 2020200343

24. Xiong Y, Sun D, Liu Y et al (2020) Clinical and high-resolution CT features of the COVID-19 infection: comparison of the initial and follow-up changes. Invest Radiol. https://doi.org/10.1097/RLI. 0000000000000674

25. Wang Y, Dong C, Hu Y et al (2020) Temporal changes of CT findings in 90 patients with COVID-19 pneumonia: a longitudinal study. Radiology. https://doi.org/10.1148/radiol.2020200843: 200843

Publisher's note Springer Nature remains neutral with regard to jurisdictional claims in published maps and institutional affiliations. 